\title{
Changing priority maps in 12- to 18-month-olds: An emerging role for object properties
}

\author{
Adam Sheya ANd Linda B. Smith \\ Indiana University, Bloomington, Indiana
}

\begin{abstract}
Everyday action in the world requires the coordination of "where," "when," and "how" with "what." In late infancy, there appear to be changes in how these different streams of information are integrated into the sequential organization of action. An experiment with 12-, 15-, and 18-month-olds was conducted in order to determine the influence of object properties and locations on the sequential selection of targets for reaching. The results reveal a developmental trend from reach decisions' being influenced only by the spatial layout of locations to the overall pattern of reaching's being influenced by the global configuration of object properties to object properties' influencing the sequential decision of what to reach to next. This trend is a new finding regarding the development of goal-directed action in late infancy.
\end{abstract}

The world is cluttered with many potential targets for action. How do we select among these targets? And, having acted, how do we select the next target? In this study, we considered the possibility that there may be fundamental changes in late infancy in how action on one target influences the choice of the next target-in particular, whether the properties of target objects, in addition to their location, first begin to play a role during this period of development.

\section{An Emerging Role for Object Properties}

In its most fundamental nature, action is about the "where," "when," and "how" and not about the "what" of object properties (Goodale \& Milner, 1992; Ungerleider $\&$ Mishkin, 1982). This is reflected in contemporary research that often distinguishes vision for action as being organized around spatiotemporal properties and vision for object recognition as being organized around the stable properties of objects (Rossetti \& Pisella, 2002). There is considerable evidence for these separate visual systems, but we know from everyday life that actions and decisions about what to do next are jointly organized by both spatiotemporal and object properties. A phenomenon concerning infants' spontaneous sequential actions on objects suggests that this coordination of what and where in the selection of targets for action may emerge when human infants are between 12 and 18 months of age.

The phenomenon is known in the developmental literature as sequential touching and is usually construed as a classificatory act (Starkey, 1981). In its usual form, infants are presented with eight objects - four from one category (e.g., cubes) and four from another (e.g., cups) - in a haphazard spatial array, and their spontaneous actions on the objects are observed (see Sugarman, 1983). With increas- ing systematicity between the ages of 12 and 18 months, infants touch perceptually similar things in sequencefor example, touching all the cups in a row (GershkoffStowe \& Namy, 1995; Mandler, Bauer, \& McDonough, 1991). Although this spontaneous sequential touching of like kinds is usually considered a form of spontaneous classificatory behavior, it may also signal fundamental change in the organization of goal-directed action-in particular, in the role of object properties in organizing the next action. This hypothesis is consistent with research in adults that shows that like objects, independent of proximity, can exert a common pull on attention (e.g., Baylis \& Driver, 1992).

\section{Priority Maps}

Action forces decision. At any moment, there may be many possible targets, but a single hand can reach to only one location at a time. The competition among potential targets (and the "decision" of where to reach next) may be understood in terms of a topographical map that represents the "salience," "relevance," or "priority" of target locations. Salience maps were originally conceived by Koch and Ullman (1985) to represent the bottom-up physical distinctiveness of objects in a visual array, but they have now been extended to encompass the dynamic integration of both top-down and bottom-up processes (Fecteau \& Munoz, 2006; Freedman \& Assad, 2006; Oristaglio, Schneider, Balan, \& Gottlieb, 2006). Both theory and empirical research with adults suggest that the activation or priority of potential targets within such a dynamic map may be modulated by factors such as task context, past events, similarity, reward, and goals, as well as physical distinctiveness (Fecteau \& Munoz, 2006; Gottlieb, 2007; Itti \& Koch, 2000; Thompson, Bichot, \& Sato, 2005). 
Within this framework, the emergence of sequential touching may be understood as an increased influence of object similarity in the dynamic updating of the priority map for target selection. During this period of development, activation in the map from touching one object may begin to spread by similarity rather than by nearness alone. Determining the accuracy of this description of developmental change would be an important first step toward an explanation of the origins of sequential touching, including the possible integration of "where" with "what."

However, because sequential touching has not been framed in these terms, there is much we do not know about the possibly relevant processes of target selection and how they might change. The present experimental design was motivated by two working hypotheses about how updating in priority maps may change with development. First, there may be an increase in dynamic updating itself. Younger infants' behavior could, in general, be less influenced by their just-previous actions, with each reach relatively independent of the previous reaches and, perhaps, more dependent on the objects themselves or on the stable properties of the array. Second, there could be a shift from dynamic updating principally in terms of space (direction of reach) to updating principally in terms of object properties. This idea that space might dominate in early priority maps fits findings that reaching is often spatially perseveratory in 8to 12-month-olds (Smith, Thelen, Titzer, \& McLin, 1999).

We developed a new task to examine these possibilities. As in sequential touching, the task presents infants with an array of eight objects of two kinds. However, the new task differs from the usual sequential-touching task so that the roles of space, of object properties, and of the configuration itself can be systematically examined. First, the eight objects were presented in fixed locations but on dampened springs, so that they jiggled when touched. In pilot work, infants consistently reached to and touched such objects, but they did not do so when the objects were rigidly attached. Second, the two categories in each array included a likely higher salient set (complex shapes: animals) and a lower salient set (simple shapes: blocks), again because past research suggested that greater attention was given to animals and/or other complex shapes (e.g., Kirchner \& Thorpe, 2006; Mandler, 2004; Sandhofer \& Smith, 2004). In this way, we asked whether the type of object has an overall influence on target selection. Third, we manipulated contrast effects in the array. Pilot studies showed the center location to be highly salient in and of itself, so we manipulated the number and location of the two kinds of objects with respect to the center. One set, which we call the target set, consisted of three objects more centrally or forwardly located; the other set, the background set, consisted of five objects, filling out the array. Although the 3:5 ratio of target to background is relatively small, the arrays (as shown in Figure 1) may be construed as a central "figure" of minority objects in a "ground." As is also shown in Figure 1, the two array configurations differ with respect to the nearness of the two objects (in the target, or minority, set) matching the center object.

\section{METHOD}

\section{Participants}

Twenty-six 12-month-olds (age range, $11.34-13.32$ months; $M=$ 12.42), twenty-three 15-month-olds (age range, 14.0-16.7 months;

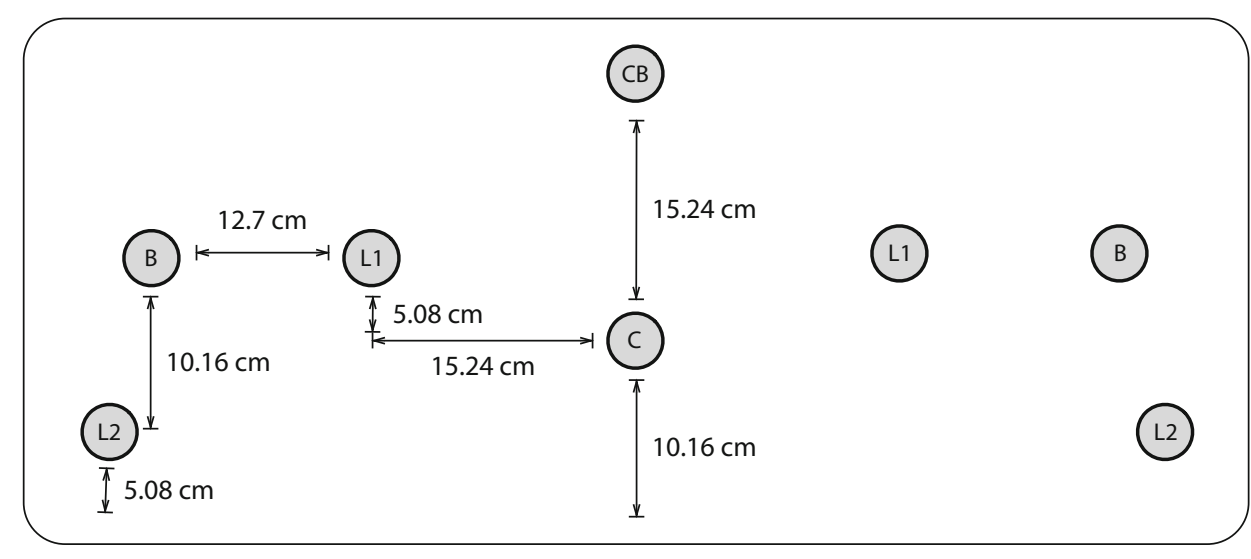

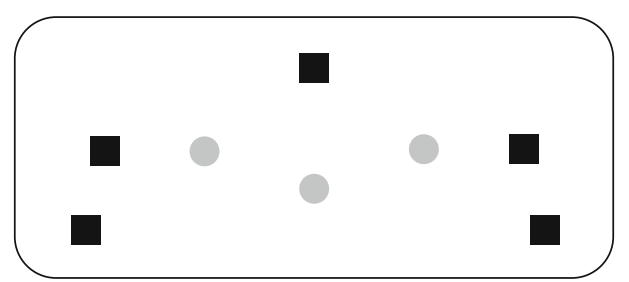

A1

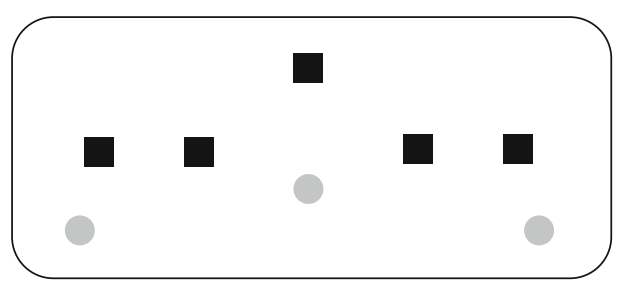

A2

Figure 1. (Top panel) Schematic of the spatial layout of the boards. (Bottom panel) The configurations of objects for Array 1 (left) and Array 2 (right). 
$M=15.26$ ), and twenty-three 18 -month-olds (age range, 16.819.2 months; $M=18.31$ ) were recruited from a middle-class population in a Midwestern town. Twelve other children were not included in the experiment due to fussiness, experimenter error, or parent noncompliance with the instructions. Participants received a small gift (e.g., t-shirt, book, small toy) in exchange for their participation.

\section{Stimuli}

Arrays consisted of eight objects ( 3.8 to $5.0 \mathrm{~cm}$ along the longest dimension) mounted by springs on a $60.96 \times 25.4 \mathrm{~cm}$ pegboard at the locations (and distances) indicated in Figure 1. Each array consisted of three identical objects of one kind and five identical objects of another kind. Every array contrasted blocks with animals. For half of the children, the minority target set was blocks; for the other half, it was animal objects. On any array, the objects of a particular kind were identical, but different arrays were constructed using different animals (turtles, lions, elephants) and blocks (cubes, spheres, cylinders) across children to ensure that any emerging patterns were not due to the particular objects used.

\section{Procedure}

Each array was presented for 2 min to an infant sitting on his or her parent's lap across a table from the experimenter. The order of presentation (of presenting either Array 1 or Array 2 first) was counterbalanced across participants. Each board was presented with the spoken instructions, "Here; you play with these." If children stopped playing before $2 \mathrm{~min}$ had elapsed, the experimenter waved her hand over the entire board, drawing the child's attention, saying, "You play with these." No other feedback or instruction was given. Parents were instructed not to direct their infant's behavior or attention in any way during the experiment. Infants' and parents' behaviors were videotaped for later coding. If a parent did not comply with the instructions, the infant's data were not included.

\section{Coding and Scoring}

Experimental sessions were coded using MacSHAPA (see Sanderson et al., 1994). Each reaching event was coded for start and completion time, the type and location of the object, and hand used. The start times were used to order the reaching events. If two reaches occurred within $1 \mathrm{sec}$ of each other with the infant not completely withdrawing the hand (e.g., patting the object), they were counted as a single reaching event. Two coders coded $30 \%$ of the data independently, and no disagreements about the order of targets or hand used were found.

\section{RESULTS}

In order to provide a clearer picture of what determines target selection, the analyses first considered factors independent of dynamic updating: location, complexity, minority/majority status, and array. We then considered the more critical question of whether and how an action influences the selection of the next target-in particular, looking at whether a next reach depends on the location of a just-previous reach or on the kind of object just reached to.

\section{Location}

Figure 2 shows the mean frequency of reaches to each location independent of the object and location of the justprevious reach. The majority of reaches went to the center location. A 3 (age) $\times 8$ (location) mixed ANOVA revealed a main effect of location $[F(7,483)=128.862, p<.001]$ but showed no other significant effects.

\section{Object and Array Effects}

By design, there are two ways through which object properties might have a persistent effect on target selection: through their frequency in the array (minority vs. majority status) or through object salience (animals vs. blocks). The appropriate contrast to test the influence of these two factors independent of the influence of location is to compare reaches to locations L1 and L2, the only locations that contained both minority and majority objects, as well as both animals and blocks, across arrays. Because there was a wide range of total reaches per array (4 to 49), the proportion of reaches was used as the dependent measure. A 3 (age) $\times 2$ (animals vs. blocks) $\times 2$ (minority vs. majority) $\times 2$ (location) mixed ANOVA on reaches to $\mathrm{L} 1$ and $\mathrm{L} 2$ revealed an effect of array $[F(1,126)=$ 20.030, $p<.001]$ and an interaction between age and array $[F(2,126)=3.655, p=.029]$ but showed no other significant effects. The effect of animal-block approached conventional standards of significance $[F(1,126)=3.362$, $p=.069]$, reflecting a greater tendency for the children to reach to L1 and L2 when those locations contained an animal $(M=.2795, S D=.1135)$ than when they contained a block $(M=.2578, S D=.1289)$. Although there were no significant interactions involving the animalblock contrast ( $p \mathrm{~s}>.11$ ), there was a greater tendency at 12 months of age to reach to animals than to reach to blocks $(M=.0475, S D=.1561)$ than at 15 months $(M=$ $.0043, S D=.1874)$ and 18 months $(M=.0098, S D=$ .1396) $[F(2,126)=1.551, p=.216]$, suggesting that the salience of individual objects might be more important early in development.

There was a slight bias at all ages for reaching to the majority objects. Figure 3 shows (and paired-sample $t$ tests with a Bonferroni correction confirmed) that only 15 -month-olds $[t(22)=3.415, p=.007]$ reached more often to a location when it contained a majority object; neither 12- nor 18-month-olds reached significantly more $(p \mathrm{~s}>.17)$ to majority objects. These results could indicate a pull toward the denser set of objects, an idea we will consider subsequently. The results also suggest that, as an effect of intrinsic salience, animal-block manipulation was not strong enough to override other factors in determining target selection. Overall, these analyses of location, object, and array effects indicate minimal developmental differences. This outcome makes conclusions from the following analyses of dynamic updating (with respect to the spatial proximity of targets and their object similarity) straightforward.

\section{Updating of Priority by Location}

Does a current reach increase the likelihood of a next reach to a near location? In the limit, this might be seen as a high rate of return to the very same location on the next reach. Accordingly, we first examined the frequency of successive reaches to the same location. A 3 (age) $\times$ 2 (array) $\times 4$ (location) mixed ANOVA revealed a difference in this tendency by age $[F(2,69)=5.359, p=.007]$, with the 15 -month-olds $(M=.2162, S D=.1367)$ showing the strongest tendency to reach back to the very same 

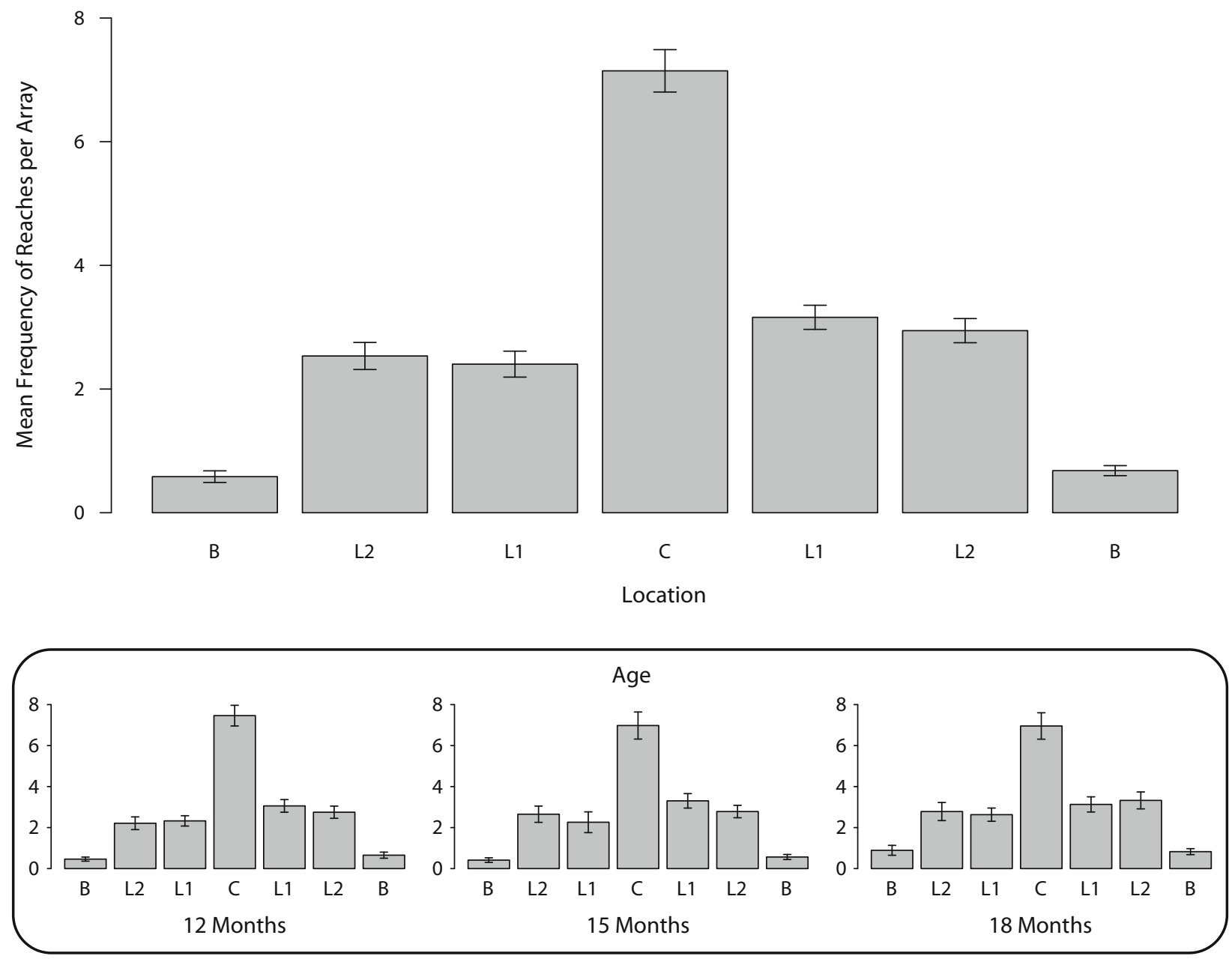

Figure 2. (Upper panel) Overall mean frequency of reaches per array to each location, except for Location $C B(M=1.236, S D=$ 0.143). (Lower panel) Means for the 12-, 15- and 18-month-olds. Bars represent standard errors.

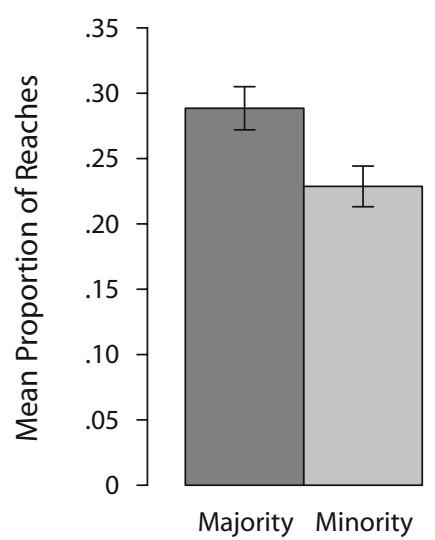

12 Months

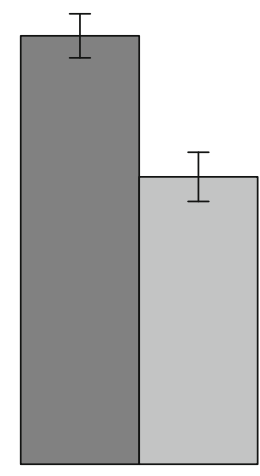

Majority Minority

15 Months

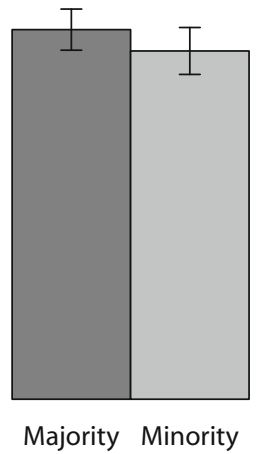

18 Months

Figure 3. Mean proportion of reaches to locations L1 and L2 when they contained majority and minority objects $\times$ age. 


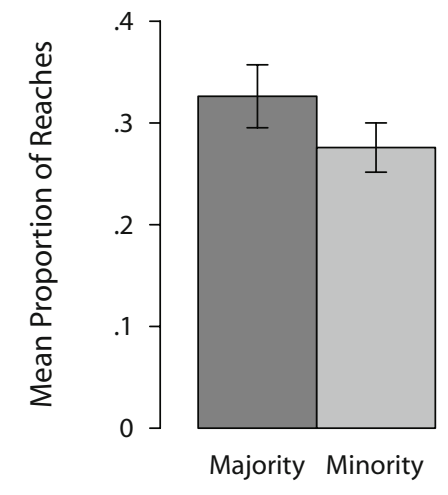

12 Months

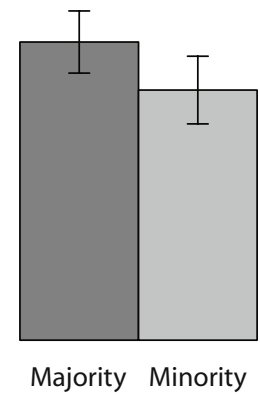

15 Months

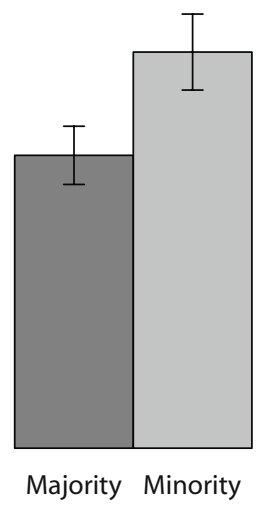

18 Months

Figure 4. Mean proportion of transitions from the center location to locations L1 and L2 when they contained minority (property match to center) and majority objects $\times$ age.

location. There was also an effect of location $[F(3,195)=$ $55.873, p<.001]$, with the strongest tendency to reach back to the same location occurring after a reach to the center location $(M=.2460, S D=.1938)$.

We also asked whether priority spreads by location. Because there is a strong bias to reach to the center, this can be answered most straightforwardly by comparing the proportion of reaches to L1 with the proportion of reaches to L2 after a reach to the center, which always contained a minority status object. A 3 (age) $\times 2$ (object) $\times$ 2 (array) $\times 2$ (location) mixed ANOVA revealed an effect of location $[F(1,69)=4.893, p=.03]$, with more reaches to L1 $(M=.3272, S D=.2127)$ than to $\mathrm{L} 2(M=.2751$, $S D=.1832)$, and an interaction between age and array $[F(2,115)=3.82, p=.025]$. No other effects approached significance $(p \mathrm{~s}>.14)$, other than a marginally significant interaction between object and minority/majority and location $[F(1,115)=3.392, p=.068]$, which was due to a greater tendency for infants to reach to animals than to blocks at L1, perhaps, again, indicating the (albeit weakly) greater salience of animals.

At all ages, children reached more often to the location adjacent to the one they last reached to, indicating that priority does spread by location. When considered independently of the just-prior reach, there was no such preference to reach to $L 1(M=.2662, S D=.1223)$ rather than to $\mathrm{L} 2(M=.2711, S D=.1215)$. The interaction between age and minority/majority status reflects a preference by 18-month-olds to reach to the L1 and L2 locations when they contained an object that was the same as the justpreviously-reached-to center object (i.e., a minority object), as indicated in Figure 4, indicating that, for this age group, target selection was influenced by object similarity.

\section{Updating of Priority by Kind}

The overall tendency for successive reaches by kind was measured by a log-odds ratio that compared the odds of reaching next to an animal object when the just-previous reach was to an animal than when it was to a block, and vice versa. The more positive the log-odds ratio, the more likely it was that a next reach went to an object with the same properties, regardless of the type of the just-reachedto object. A 3 (age) $\times 2$ (array) mixed ANOVA revealed that 18 -month-olds $(M=0.155, S D=1.175)$ were more likely to next reach to an object with the same properties than were 15 -month-olds $(M=-0.253, S D=1.366)$ or 12-month-olds $(M=-0.704, S D=1.082)[F(2,69)=$ $4.749, p=.012]$. There were no other significant effects $(p s>.07)$. These results suggest an increase in the role of object properties in organizing sequential action. Analyses of the proportion of transitions to a new location that contained the same object kind yielded the same conclusion. A 3 (age) $\times 2$ (array) mixed ANOVA revealed a significant effect of age $[F(2,69)=7.561, p=.001]$, with the 18 -month-olds $(M=.4304, S D=.1512)$ reaching next to the same object type more often than did 15-month-olds $(M=.3034, S D=.1415)$ or 12-month-olds $(M=.3295$, $S D=.1614)$. There were no other significant effects $(p s>.2)$. Again, there is a clear increase in influence of object kind on sequential reaches with age; this appears particularly markedly in 18-month-olds.

To test the strength of these observations, we also considered reaches to L1 and L2 after a reach to the center, as a function of array, because these two locations provide a direct contrast of location and kind effects. This comparison was performed in the 3 (age) $\times 2$ (animal vs. block) $\times 2$ (minority/majority) $\times 2$ (location) mixed ANOVA presented in the "Updating of Priority by Location" section above. A significant interaction between age and minority/majority was found, indicating, as is shown in Figure 4, that the 18-month-olds reached to a location (either L1 or L2) more often when it contained the same kind of object than when it contained a different kind of object, whereas the younger infants did not. Interestingly, the same pattern was observed when the transitions were in the reverse direction, from L1 or L2 to the center. Next reaches from either location went to the center more often when the location contained a minority object $(M=$ $.4599, S D=.2740)$ than when it contained a majority object $(M=.3256, S D=.1714)[t(21)=2.70, p=.013]$. Again, there is a clear effect of kind on the selection of targets during sequential reaching for 18 -month-olds. The 
two younger groups of infants showed no significant effects of kind in parallel analyses $(p \mathrm{~s}>.08)$.

In sum, the younger infants' sequential reaching demonstrated no sensitivity to the visual similarity of targets. The infants did, however, demonstrate sensitivity to the spatial configuration of the object array. Overall, they reached more to the center location and, after a reach to the center, more often to a nearby location, both when it contained the same object as the center and when it contained an object different from the center. In contrast, reaches by 18-month-olds increased the priority of objects identical to the object just reached to, indicating an increase in priority spread by similarity.

\section{DISCUSSION}

These findings present new evidence regarding two fundamental issues: (1) the sequential organization of behavior, including how a just-past action potentiates the next action (Lashley, 1951); and (2) the integration of sensory information for guiding movement (Churchland \& Sejnowski, 1994). Research in a number of domains, including search tasks (Treisman \& Gelade, 1980) and tracking tasks (Blaser, Pylyshyn, \& Holcombe, 2000; see also Carey \& Xu, 2001), as well as in neuropsychology (Desimone \& Duncan, 1995) and cognitive neuroscience (Goodale \& Milner, 1992; Tanné, Boussaoud, Boyer-Zeller, \& Rouiller, 1995), suggest that information about object location and information about object properties involve separate processing streams. But everyday behavior requires us to coordinate these two sources of information to achieve coherent sequences of behaviors. The present results suggest that this coordination may strengthen between 12 and 18 months, so that the sequence of reaches by the youngest infants appears to be governed primarily by the location of the objects, without regard to their perceptual properties. For the oldest children, in contrast, reaching priority spreads by the similarity of the targets, increasing the likelihood of sequential reaching to like things.

The pattern by the older children is in accord with sequential touching patterns often interpreted as classificatory acts (Mandler et al., 1991). That interpretation and the present one, in terms of a priority map, are not at odds. A priority map that spreads activation by the similarity of object properties and not just by spatial proximity is a map that organizes potential targets by kind. However, demonstrating the recognition of like kinds through sequential touching also requires actions that coordinate location and object information. Thus, the present conceptualization of this phenomenon in terms of a priority map may contribute to a deeper understanding of how and why sequential touching of like kinds emerges when it does. At present, the only explanation of this phenomenon is that infants sequentially touch like kinds because they recognize their similarity. This is not an adequate explanation, because considerable research using preferential looking as a dependent measure shows that much younger children recognize like kinds (Quinn \& Eimas, 1998). The influence of object similarity on action requires the coordination of vision for action and vision for object recognition into one integrative priority map for object-directed action, and it may be this development that gives rise to the sequential touching of like kinds in late infancy.

From this perspective, the 15-month-olds present an intriguing intermediate stage in the integration of information about object properties into the coordination of sequential action. At all ages, there was a slight bias to reach more to majority than to minority objects, but this was particularly strong (and highly reliable) for the 15-month-olds. It may be that object similarities first influence action via an overall contextual salience, perhaps on the basis of the frequency of object properties in the array. The global layout of properties across locations may be compared, creating greater priority for the more frequent kind. In this way, target selection can be influenced by the object properties across the array but not by the object properties of the specific object just reached to. The interesting developmental question is how this global influence of object properties leads to object properties influencing the dynamic updating of the priority map.

In conclusion, the present task presents new findings on the early development of sequential goal-directed action and raises new questions about the integration of object properties into action. Relevant future questions concern how extrinsic salience, contrast, location, and history with the particular objects may interact at each developmental level, how the dynamics of priority maps prior to 12 months and after 18 months are changing, and the mechanisms of this change.

\section{AUTHOR NOTE}

This research was supported by R01 HD28675 from NICHHD. Correspondence concerning this article should be addressed to A. Sheya, who is currently a postdoctoral fellow at the Infant Development Research Center, Florida International University, 11200 S.W. 8th Street, University Park, DM116, Miami, FL 33199 (e-mail: asheya@fiu.edu).

\section{REFERENCES}

BAYlis, G. C., \& Driver, J. (1992). Visual parsing and response competition: The effect of grouping factors. Perception \& Psychophysics, 51, 145-162.

Blaser, E., Pylyshyn, Z. W., \& Holcombe, A. O. (2000). Tracking an object through feature space. Nature, 408, 196-199.

CAREY, S., \& XU, F. (2001). Infants' knowledge of objects: Beyond object files and object tracking. Cognition, 80, 179-213.

Churchland, P. S., \& Sejnowski, T. J. (1994). The computational brain. Cambridge, MA: MIT Press.

Desimone, R., \& Duncan, J. (1995). Neural mechanisms of selective visual attention. Annual Review of Neuroscience, 18, 193-222.

Fecteau, J. H., \& Munoz, D. P. (2006). Salience, relevance, and firing: A priority map for target selection. Trends in Cognitive Sciences, 10, 382-390.

Freedman, D. J., \& Assad, J. A. (2006). Experience-dependent representation of visual categories in parietal cortex. Nature, 443, 85-88.

Gershroff-Stowe, L., \& Namy, L. L. (1995, March). Sequential touching and spatial grouping: A curvilinear developmental trend. Paper presented at the 62nd Anniversary Meeting of the Society for Research in Child Development, Indianapolis, Indiana.

Goodale, M. A., \& Milner, A. D. (1992). Separate visual pathways for perception and action. Trends in Neurosciences, 15, 20-25.

GotTlieb, J. (2007). From thought to action: The parietal cortex as a bridge between perception, action, and cognition. Neuron, 53, 9-16.

IтTі, L., \& Koch, C. (2000). A saliency-based search mechanism for overt and covert shifts of visual attention. Vision Research, 40, 1489 1506. 
Kirchner, H., \& Thorpe, S. J. (2006). Ultra-rapid object detection with saccadic eye movements: Visual processing speed revisited. Vision Research, 46, 1762-1776.

Koch, C., \& Ullman, S. (1985). Shifts in selective visual attention: Towards the underlying neural circuitry. Human Neurobiology, 4, 219-227.

LASHLEY, K. S. (1951). The problem of serial order in behavior. In L. A. Jeffress (Ed.), Cerebral mechanisms in behavior (pp. 112-136). New York: Wiley.

MANDLER, J. M. (2004). The foundations of mind: Origins of conceptual thought. New York: Oxford University Press.

Mandler, J. M., Bauer, P. J., \& McDonough, L. (1991). Separating the sheep from the goats: Differentiating global categories. Cognitive Psychology, 23, 263-298.

Oristaglio, J., Schneider, D. M., Balan, P. F., \& Gottlieb, J. (2006). Integration of visuospatial and effector information during symbolically cued limb movements in monkey lateral intraparietal area. Journal of Neuroscience, 26, 8310-8319.

QuinN, P. C., \& Eimas, P. D. (1998). Evidence for a global categorical representation of humans by young infants. Journal of Experimental Child Psychology, 69, 151-174.

Rossetti, Y., \& Pisella, L. (2002). Several "vision for action” systems: A guide to dissociating and integrating dorsal and ventral functions (tutorial). In W. Prinz \& B. Hommel (Eds.), Attention and performance XIX: Common mechanisms in perception and action. (pp. 62119). Oxford: Oxford University Press.

Sanderson, P., Scott, J., Johnston, T., Mainzer, J., Watanabe, L., \& JAMES, J. (1994). MacSHAPA and the enterprise of exploratory sequential data analysis (ESDA). International Journal of HumanComputer Studies, 41, 633-681.

Sandhofer, C. M., \& Smith, L. B. (2004). Perceptual complexity and form class cues in novel word extension tasks: How 4-year-old children interpret adjectives and count nouns. Developmental Science, 7, 378-388.

Smith, L. B., Thelen, E., Titzer, R., \& Mclin, D. (1999). Knowing in the context of acting: The task dynamics of the A-not-B error. Psychological Review, 106, 235-260.

STARKEY, D. (1981). The origins of concept formation: Object sorting and object preference in early infancy. Child Development, 52, 489-497.

Sugarman, S. (1983). Children's early thought: Developments in classification. New York: Cambridge University Press.

Tanné, J., Boussaoud, D., Boyer-Zeller, N., \& Rouiller, E. M. (1995). Direct visual pathways for reaching movements in the macaque monkey. NeuroReport, 7, 267-272.

Thompson, K. G., Biснот, N. P., \& Sato, T. R. (2005). Frontal eye field activity before visual search errors reveals the integration of bottomup and top-down salience. Journal of Neurophysiology, 93, 337-351.

Treisman, A. M., \& Gelade, G. (1980). A feature-integration theory of attention. Cognitive Psychology, 12, 97-136.

Ungerleider, L. G., \& Mishrin, M. (1982). Two cortical visual systems. In D. J. Ingle, M. A. Goodale, \& R. J. W. Mansfield (Eds.), Analysis of visual behavior (pp. 549-586). Cambridge, MA: MIT Press.

(Manuscript received October 1, 2008; revision accepted for publication September 9, 2009.) 